\section{Recent Activities In Environmental Microscopy}

James R. Millette

MVA, Inc., Norcross GA

The microscope has always played an important part in the field of environmental science. Initially it was involved mostly with those who analyzed for biological indicators of pollution or those who examined air pollution particles, but more recently it is being used by a large number of analysts of particulate samples following the concern over asbestos in schools. With the Asbestos Hazard Emergency Response Act (AHERA) ${ }^{1}$ of 1987, transmission electron microscopy (TEM) formally joined polarized light microscopy (PLM) and phase contrast microscopy (PCM) as a recognized regulation-required standard procedure for the analysis of asbestos. While asbestos remains a considerable activity in environmental microscopy, other areas including analysis of non-asbestos fibers such as ceramic fibers, lead particle source determination, respirable silica measurement, and indoor air quality particle examination have seen some interesting developments.

Standard methods for fibers other than asbestos are being considered. Methods for determining the concentrations of airborne silicon carbide whiskers or ceramic fibers by scanning electron microscopy (SEM). TEM, and PCM have been balloted under the American Society for Testing and Materials $(\text { ASTM) })^{2}$ Committee D-22 on Sampling and Analysis of Atmospheres this past summer. Papers on the subject of microscopy studies of fibrous glasses and manmade vitreous fibers are planned for the next Environmental Information Association (EIA) meeting to be held in San Diego in the spring of 1994

Methods for the computer-controlled SEM are being used in conjunction with $x$-ray diffraction to help producers of minerals containing silica, such as clays, talc, pumice, sand \& gravel, perlite, mica, calcium carbonate and bauxite, gain information about the particle size of the silica particles.

Microscopy is also being used in the environmental area of lead contamination. Papers describing the use of the SEM in the characterization of house dust as part of a lead abatement clean-up process and the speciation of lead in urban soils by computer assisted SEM/EDS were presented at the 1993 ASTM Boulder Conference on Lead in Paint, Soil, and Dust which was held July $25-29$ at the University of Colorado. This conference was sponsored by ASTM Committees D-22 on Sampling and Analysis of Atmospheres, E- 6 on Performance of Buildings, D-1 on Paint and Related Coatings, Materials and Applications and D-18 on Soil and Rock. It was clear at the meeting that microscopy will play an important role in determining the source of lead in dusts.

There appears to be renewed interest in the analysis of airborne particulate samples collected in conjunction with studies of indoor air quality Pollen identification was the subject of a State Microscopical Society of Illinois workshop last spring and will be the topic of a presentation to be given at the Environmental Information Association meeting next spring.

Currently there are four standard methods involving the analysis of asbestos by TEM under consideration by the ASTM Committee D-22 on Sampling and Analysis of Atmospheres. Two TEM methods involve the analysis for asbestos in settled dust. One utilizes a microvacuuming procedure and an indirect preparation procedure while the other uses an adhesive sampler and a direct preparation procedure. The third method being considered is for passive dust collection and analysis by TEM. The fourth method is a TEM method for ambient air analysis which is the version of the International Standards Organization (ISO) proposed method in ASTM format. A PLM method for the analysis of bulk samples for asbestos is currently being circulated in the ASTM gray pages. Although not yet required under AHERA, the EPA has a new publication ${ }^{3}$ describing the PLM procedure for bulk samples. This 87 page Test Method includes information about PLM and X-ray diffraction analysis of asbestos - containing material which has been gained over the last few years.

Asbestos in drinking water is also an issue for environmental microscopy. In 1992 the USEPA, under the Safe Drinking Water Act, published a regulation in the Federal Register which specified that most drinking water supplies had to be tested for asbestos and that the analysis of public drinking water supplies for asbestos shall be conducted by transmission electron microscopy. The method required was a 1983 EPA publication. ${ }^{5}$ Because the regulation was witten almost ten years after the method was published, a peer review panel was convened to review the method and provide guidance as to acceptable changes in the protocol. A clarification memo was released by EPA in March 1993 which provides guidance for some of the steps in the procedure to make the method specifically applicable to the drinking water analysis which only requires measurement of asbestos fibers over $10 \mu \mathrm{m}$ in length. ${ }^{6}$ The following are some of the key items in the document. The Maximum Contaminant Level $(\mathrm{MCL}$ ) is 7 million fibers per liter (MFL) of asbestos fibers greater than $10 \mu \mathrm{m}$ in length. The analyst is directed to achieve an analytical sensitivity of 200,000 fibers per liter $(0.2 \mathrm{MFL})$. Samples may be analyzed at $10,000 \mathrm{X}$ for counting fibers over $10 \mu \mathrm{m}$ in length. States may choose to count fibers less than $10 \mu \mathrm{m}$ in length. Amphibole asbestos must be identified by both electron diffraction and quantitative $x$-ray analysis. The American Water Works Association (AWWA) has recently completed balloting for the 18th Edition Supplement of the Standard Methods for the Analysis of Water and Waste Water. The method for the analysis of asbestos in water (Section 2570) passed with no negative ballots.

\section{References:}

1. Federal Register 40 CFR Part 763, Asbestos-Containing Material in Schools; Final Rule and Notice Oct. 30, 1987.

2. ASTM 1916 Race Street, Philadelphia, PA 19103-1187. 215-299-5400

3. Perkins, R.L. and Harvey, B.W. Test Method (Method for the determination of asbestos in bulk building materials) (EPA/600/R-93/116, July, 1993)

4. Federal Register 5731838 , Section 141.23(k) (4) July 17, 1992

5. Analytical Method for the Determination of Asbestos Fibers in Water (EPA-600/4-83)

6. Memo from James M. Conion, Office of Ground Water and Drinking Water, USEPA, Washington DC March 9,1993. (Details can be found in an article to be published soon in the Environmental Information Association Journal)
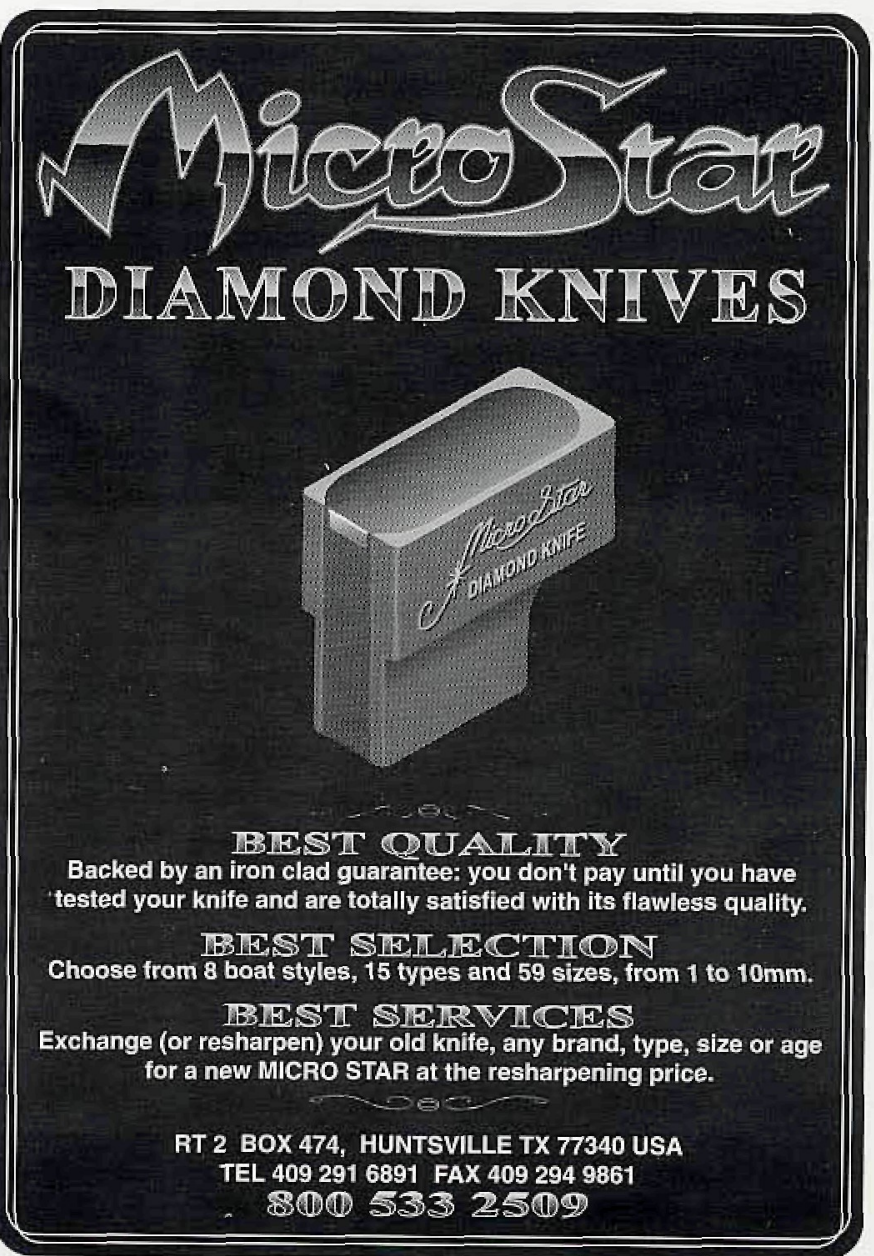